\title{
Estimation of ground reaction forces and ankle moment with multiple, low-cost sensors
}

\author{
Daniel A. Jacobs (D) and Daniel P. Ferris (D)
}

\begin{abstract}
Background: Wearable sensor systems can provide data for at-home gait analyses and input to controllers for rehabilitation devices but they often have reduced estimation accuracy compared to laboratory systems. The goal of this study is to evaluate a portable, low-cost system for measuring ground reaction forces and ankle joint torques in treadmill walking and calf raises.

Methods: To estimate the ground reaction forces and ankle joint torques, we developed a custom instrumented insole and a tissue force sensor. Six healthy subjects completed a collection of movements (calf raises, $1.0 \mathrm{~m} / \mathrm{s} \mathrm{walking}$, and $1.5 \mathrm{~m} / \mathrm{s}$ walking) on two separate days. We trained artificial neural networks on the study data and compared the estimates to a multi-camera motion system and an instrumented treadmill. We evaluated the relative strength of each sensor by testing each sensor's ability to predict the ankle joint torque calculated from a reference inverse kinematics algorithm. We assessed model accuracy through root mean squared error and normalized root mean square error. We hypothesized that the estimation of the models would have normalized root mean square error measures less than $10 \%$.

Results: For walking at 1.0 and walking at $1.5 \mathrm{~m} / \mathrm{s}$, the single-task, intra-day and multi-task, intra-day predictions had normalized root mean square error less than $10 \%$ for all three force components and both center of pressure components. For the calf raise task, the single-task, intra-day and multi-task, intra-day predictions had normalized root mean square error less than $10 \%$ for only the anterior-posterior center of pressure. The multi-task, intra-day model had similar predictions to the single-task, intra-day model. The normalized root mean square error of predictions from the insole sensor alone were less than $10 \%$ for walking at $1.0 \mathrm{~m} / \mathrm{s}$ and $1.5 \mathrm{~m} / \mathrm{s}$. No sensor was sufficient for the calf raise task. The combination of the insole sensor and the tendon sensor had lower normalized root mean square error than the individual sensors for all three tasks.
\end{abstract}

Conclusions: The proposed sensor system provided accurate estimates for five of the six components of the ground reaction kinetics during walking at 1.0 and $1.5 \mathrm{~m} / \mathrm{s}$ and one of the six components during the calf raise task. The normalized root mean square error of the predictions of the ground reaction forces were similar to published studies using commercial devices. The proposed system of low-cost sensors can provide useful estimations of ankle joint torque for both walking and calf raises for future studies in mobile gait analysis.

Keywords: Locomotion, Gait, Biomechanical analysis, Plantar pressure insole, Ground contact, Sensor fusion

*Correspondence: jacobsda@umich.edu

School of Kinesiology, University of Michigan, 401 Washtenaw Ave CCRB, Ann Arbor, MI, USA 


\section{Background}

Increasing health care costs and the need for more convenient and cost-effective patient care are driving investigations into research and development of mobile health monitoring systems. Advances in wearable technologies, as one subgroup of mobile health monitoring technologies, can enable more affordable and accessible health care by developing low-cost, unobtrusive measurement devices that can provide real-time feedback to patients and health care providers on the patient's health in their every day lives $[1,2]$. The timely measurement and communication between wearable technologies, patients, and health care providers could have significant effects on the quality of life of patients by helping drive health care from treatment to prevention [3].

Estimating the ground reaction forces and joint moments of humans in the real world could have substantial clinical impact by providing assessments of pathological gait, fall detection in the elderly, and biofeedback data for home interventions. Currently, the gold-standard of clinical gait analysis using motion capture and force plates can estimate ground reaction forces and joint moments without invasive sensors. However, the viability of gait analysis is restricted due to their size, cost, and laboratory size. The aim of this study was to test the ability of multiple sensors to provide low-cost measurements of ground reaction forces and ankle joint moments.

Many wearable sensors have difficulty providing robust estimations in the presence of substantial movement variability. Studies on wearable sensors that only investigate overground and treadmill walking do not deal with the amount of variability that happens in daily life [4-8]. In some cases, the investigated sensor is tested only on the stance phase of walking $[9,10]$. Investigating only walking leads to overestimation of sensor accuracy due to the fact that the passive dynamics of walking lead to stereotypical patterns that are easier to predict. Not including swing phase data in estimation also reduces variability in the data set because it assumes that nonlinear transition between stance and swing is predicted perfectly despite sensor noise.

Investigating non-stereotypical tasks can lead to more accuracy assessments of wearable technology. One nonstereotypical task that could be included in performance testing of wearable technologies is the calf raise. Biomechanically, calf raises are interesting because the major functional behavior is balancing rather than forward propulsion like in walking. The profiles of ground reaction force and center of pressure during a balancing task have larger variance than a stereotypical task which makes accurate prediction more challenging. Clinically, calf-raises are often elements of lower limb rehabilitation protocols following Achilles tendon and anterior cruciate ligament injury $[11,12]$ and training protocols for improving balance and gait stability [13].

We evaluated a system consisting of two custom, lowcost sensors: a custom plantar pressure insole and a non-invasive tendon load cell. By fusing multiple sensors, the estimation accuracy of the sensor system could be reduced enough to create an acceptable, generalizable model that is robust and repeatable across different tasks. Several groups have demonstrated that plantar pressure insoles provide sufficient data to estimate ground reaction forces (GRF), center of pressure (COP), and ankle joint torques (AJT) $[5,6,8]$ yet insole performance on nonstereotypical tasks is unclear. Tendon sensors, such as tendon buckles $[14,15]$, non-invasive strain sensors [16], and ultrasonic velocity measurements [17] have also provided useful data on muscle and tendon state but most sensors are bulky and require specialized equipment that is not mobile.

In addition to task variability, sensor characteristics, such as drift and creep, can negatively affect estimation with insoles [18-20]. Studies on insole sensor variability and repeatability have shown that current devices need calibration in order to perform adequately [21, 22] and that long term performance is still an issue [21, 23-26].

In this study, we quantified the predictive ability of the sensors to estimate the ground reaction forces, center of pressure, and ankle joint torques during normal walking and calf-raises in healthy young adults. We collected steady state treadmill walking at two speeds and a set of five self-paced calf raises. To evaluate long-term performance, we collected trials on two different days without external calibration of the sensor or fitting. We built artificial neural network regression models to estimate the ground reaction forces, center of pressure, and ankle joint torques from the prototype sensor data and compared the predictions to the reference data from motion capture and instrumented force plates. To assess model accuracy, we performed a series of estimations on withheld data and calculated the mean performance measures across intra-day, interday, single-task, and multi-task groupings. We used the root mean square error (RMSE) and the normalized root mean square error (NRMSE) as measures of model performance.

\section{Methods}

\section{Subjects}

Six healthy subjects (2 Female, 4 Male) participated in this study: (mean \pm std) age $24.5 \pm 3.6$ years, height $1.78 \pm 0.07 \mathrm{~m}$, leg length $0.94 \pm 0.05 \mathrm{~m}$, and mass $69.9 \pm 12.64 \mathrm{~kg}$. Each collection day, the subjects performed three trials of walking at $1.0 \mathrm{~m} / \mathrm{s}$, walking at $1.5 \mathrm{~m} / \mathrm{s}$, and three sets of five calf raises. Each subject performed two collections spaced $40 \pm 15$ days apart. 


\section{Ethics, consent and permissions}

All subjects gave written informed consent in accordance with the Declaration of Helsinki. The Institutional Review Board of the University of Michigan (FWA \#00004969) approved the study protocol.

\section{Data collection}

We recorded marker data at $100 \mathrm{~Hz}$ with a 10 camera motion capture system (Vicon, Inc. USA). We collected ground contact forces at $1 \mathrm{kHz}$ with an instrumented split-belt treadmill (Bertec Inc. USA). A data acquisition system (dSPACE GmhB, Germany) running Real-Time Workshop (Mathworks Inc., USA) captured the voltage signals from the insole and tendon sensors at $1 \mathrm{kHz}$. We filtered the ground contact forces and moments from the instrumented force plates and the voltage signals from the sensors with a low-pass, fourth order butterworth filter at $25 \mathrm{~Hz}$. We synchronized the data acquisition system and the motion capture system by co-recording a manuallytriggered square wave from a signal generator in both systems. The data streams were aligned by edge detection of the square wave in the post-processing routines.

In a few trials, an intermittent break in the electrical connection added artifact noise to the sensor data. The noise did not strongly contaminate the data and we did not filter the artifact or exclude any data from our analysis.

\section{Sensing hardware}

During the study, each subject wore an orthopedic shoe with a custom insole insert to measure localized changes in plantar pressure. Each insole contained eight custom neoprene bladders instrumented with miniature, amplified, temperature-compensated pressure sensors (Honeywell, Inc. USA, SSCDANN030PGAA5). The pneumatic bladders had a loading area of $25.4 \times 25.4 \times 6.35 \mathrm{~mm}$ and a wall thickness of $1.58 \mathrm{~mm}$. The total cost of a pair of instrumented shoes was $\$ 800$ (Sensors: $\$ 512$, Bladders: \$144, PCB \$132, insole foam, glue, and misc: \$10). The base weight of the shoe was $480.2 \mathrm{~g}$ and total weight of the sensors was $257.8 \mathrm{~g}$ which accounted for an increase of 54 percent over normal attire (Table 1 ).

Table 1 Mass values of a single side of sensor system. The total weight of the insole sensors, Achilles tendon sensors, and circuitry increased the base weight of the shoe by $54 \%$

\begin{tabular}{ll}
\hline Part & Mass \\
\hline Pressure Sensors and Circuitry & $137 \mathrm{~g}$ \\
Achilles Tendon Sensor & $44 \mathrm{~g}$ \\
Bladder & $10 \mathrm{~g}$ \\
Sensor Subtotal & $258 \mathrm{~g}$ \\
One Shoe (US Men's 10) & $480 \mathrm{~g}$ \\
Total Weight & $738.0 \mathrm{~g}$ \\
\hline
\end{tabular}

Each subject also wore a miniature beam load cell on the distal end of the tibia above the calcaneus to measure localized tissue forces around the Achilles tendon. The tendon sensor consisted of a thin film load cell (Strain Measurement Devices, Inc. USA, S100) connected to base aluminum block and a delrin tendon cup. The total cost for the pair of the tendon sensors was $\$ 340$ (Sensors $\$ 330$, misc aluminum and delrin \$10).

Both devices are simple to manufacture with standard laboratory tools and they can be adjusted for different users easily. Figure 1 shows several photos of the individual components and the full system worn by subject. Figure $1 \mathrm{a}$ and $\mathrm{b}$ show the insole (without the top comfort foam layer) with the bladders inserted. Figure 1e shows the bladder and custom printed circuit board for the pressure sensors. Figure 1c shows a tendon sensor along with two acetal resin tendon cups to assist in fitting different subjects. Figure 1d shows the complete system on a subject. We affixed the tendon sensor to the subject with double sided tape, then secured the entire sensor in flexible athletic tape.

\section{Analysis}

We calculated the ground reaction forces (GRF), center of pressure (COP) and vertical torque in the laboratory coordinate system. The center of pressure and the vertical torque components of the ground reaction forces were set to zero when the vertical force measurement was less then $5 \%$ of body weight.

We used a 23 degree of freedom musculoskeletal model (gait2354) in the open-source software OpenSim [27] for our analysis. Using the marker and treadmill data, we scaled the model and ran inverse kinematics and inverse dynamics algorithms to calculate the generalized coordinates and generalized forces of the model in each trial. We transformed the location of the center of pressure from the laboratory coordinate system to a foot fixed coordinate system with the origin in the calcaneus to normalize the subjects steps and remove the effect of treadmill position.

For the mean component profiles comparison, we normalized each subject's step to gait cycle phase or task phase. We normalized the center of pressure locations as a percentage of the leg length $(\% \mathrm{~L})$, forces as a percentage of body weight $(\% \mathrm{BW})$, and moments as a percentage of the product of body weight and leg length (\% BWL). We estimated the leg length of the subject from the mean of the left and right greater trochanter $\mathrm{z}$ markers during the standing trials.

For the prediction of the GRF, we calculated the root mean square error (RMSE) and the normalized root mean square error (NRMSE). The NRMSE was calculated by dividing the RMSE by the range of the signal 


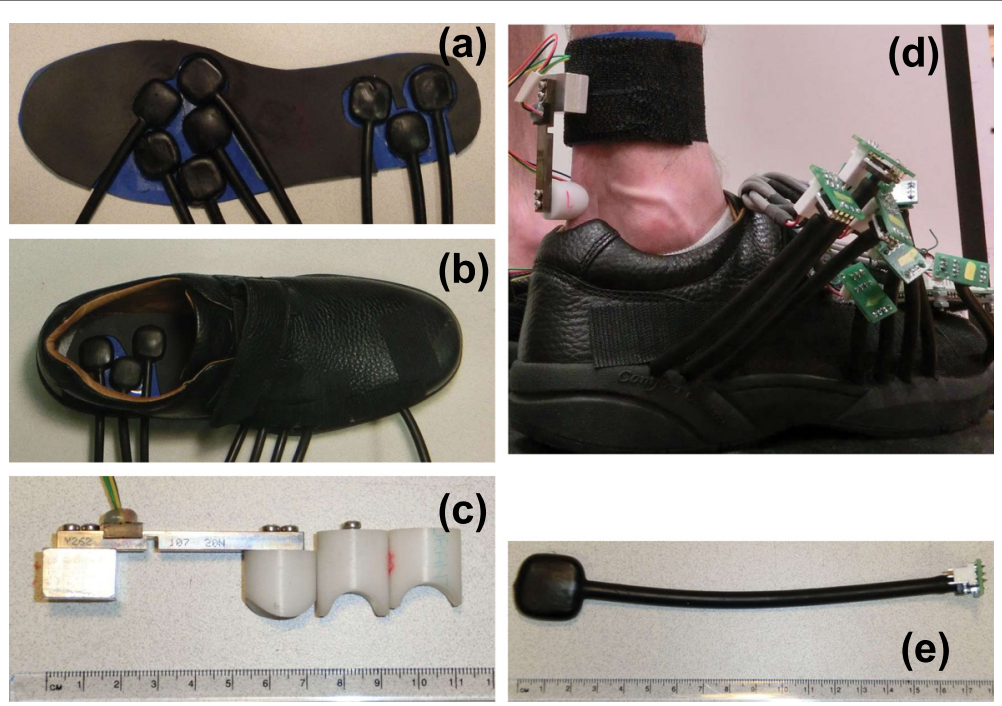

Fig. 1 Hardware photos. The collage shows photos of a the insole and inlaid pressure sensors (top foam layer missing) $\mathbf{b}$ the insole embedded in the orthotic shoe, c a miniature beam load cell and the plastic tendon cups for the tendon sensor, $\mathbf{d}$ a subject wearing both of the sensors and e the custom pressure sensor and amplifier unit. The total cost of a pair of insoles was $\$ 800$ and the total cost of the pair of load cells was $\$ 420$. Both sensors were easily constructed, assembled, and disassembled without specialty tools

data in the reference signal from the motion capture and instrumented force plate data. For the prediction of the AJT, we also compared the relative strength of the individual sensors in the sensor set by calculating the accuracy for combinations of sensors. As a feature set, we compared the individual ankle angle estimated from the motion capture data, the voltage from the tendon sensor, and the vertical force and fore-aft center of pressure estimated from the insole sensor.

Although the true measure of accuracy for clinical validity is unknown, we set the accuracy cutoff at $10 \%$ for this study to match the reported accuracy (5-28\%) of studies on walking that used commercial pressure and insole mats as those devices are utilized in clinics.

\section{Regression models}

We created a series of single-hidden layer, 10 node, feedforward neural networks in Matlab. Each model was tested on data withheld from the training phase. We used a cross-fold validation scheme to create a series of models for each subject and calculated the mean model accuracy across the iterations [28].

Splitting data for regression models can be done supervised or unsupervised depending upon the experimental data [29]. We chose a supervised method where we split the training and test data into groups of intra-day and inter-day sets in order to ask interpretable questions, e.g., how accurate is the model likely to be when tested on subjects within a testing day or on a new testing day.
It is important to split the data into sections where the regions of prediction are equivalent to avoid local information and improve the bias properties of the predictors [29]. Splitting the data into intra-day and inter-day sets creates equivalent prediction areas because each set contains an equal number of cycles and the range and variance of the signal is similar. Splitting the data into randomized sections could result in an uneven split of the gait cycle which would have different range and variance properties which could bias the estimate of model accuracy.

From the data set, we created the following groups:

- Single-Task, Intra-Day

- Single-Task, Inter-Day

- Multi-Task, Intra-Day

Our training and testing groups are represented visually in Fig. 2 in a grid representation. For the single-task sets, each row represents a set of tests, where the green boxes indicates the trial used for training and the blue boxes represent the trials used for testing.

For example, the single-task, intra-day set consisted of testing each trial on a set of withheld data consisting of the remaining two trials in that day e.g the model trained on the first trial was tested on the data from the second and third trials on that day and the model trained on the second trial was tested on the data from the first and third trials on that day.

In the single-task, intra-day, the grid coloring in Fig. 2 indicates that for each of the 6 models (green boxes) 

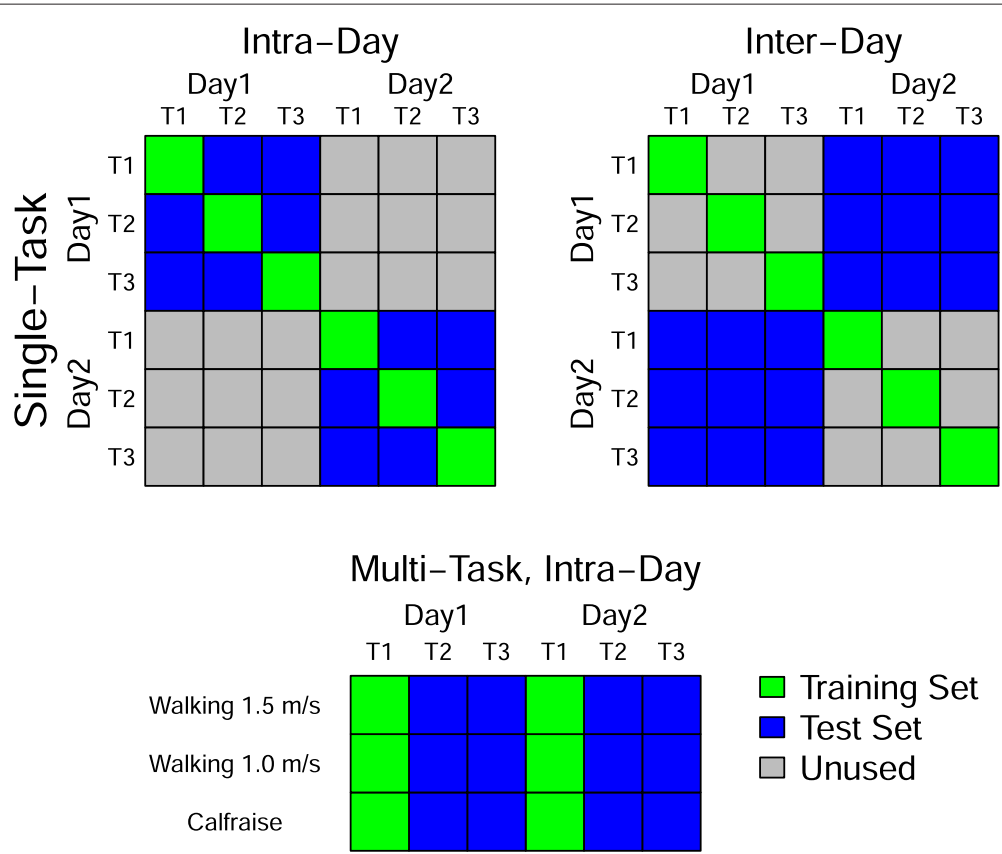

Fig. 2 Analysis group illustration. On each collection day, we collected three trials of each task for a total of six trials. (TOP) In the single-task groups, we formed a six-by-six grid of training and test data sets for each task. The top three grids show a graphical representation of the three single-task groupings. The green squares represent the trial from which the training and cross validation data were taken and the blue squares represent the test sets which were averaged for the results. Note that union of the blue squares represents the complete grid with each set used once. (BOTTOM) For the multi-task group, we created a data set of eighteen trials from the three trials on both days for the three tasks. We created a training set using the first trials on both days for all three tasks and a test set from the second and third trials on both days

we produced 12 test performance measures (blue boxes) yielding a final performance metric that was the average of 120 values (10 subjects, 12 tests) for each kinetic component, task, and subject.

The multi-task, intra-day group, consisted of a single model trained on the first trial of each task from day 1 and day 2 and tested on the remaining two trials from each day (6 training trials and 12 testing trials). The final performance metric for each component was also the average of 120 values (10 subjects, 12 tests) for each kinetic component.

The neural network training algorithms further divided the input data into subgroups allocated as: $70 \%$ training, $15 \%$ validation, and $15 \%$ test. To avoid overfitting the data, the Matlab training algorithm halted the training when the performance decreased on the validation data set after an iteration. The output of each individual training on the remaining $15 \%$ test data was discarded in favor of the output from the grid tests.

For each model, we calculated the root mean square error (RMSE) and the normalized root mean square error (NRMSE) on the withheld test data. For the final reported model performance, we calculated the mean RMSE and NRMSE across all subjects.

\section{Results}

For walking at $1.0 \mathrm{~m} / \mathrm{s}, 1.5 \mathrm{~m} / \mathrm{s}$ and calf raises, the differences between the mean ground reaction kinetics for the reference (solid black line) and estimated (blue lines) were small (Fig. 3). The differences between the reference and estimated curves are greatest for the single-task, inter-day subgroup.

The normalized root mean square error for the singletask, inter-day group was than the single-task intraday group for all components and tasks (Fig. 4 and Table 2). The multi-task, intra-day group had mean normalized root mean square error lower than the singletask, inter-day group for all components and tasks except for the anterior-posterior force component of the calf raise task.

For walking at $1.0 \mathrm{~m} / \mathrm{s}$, the single-task, intra-day, the single-task, inter-day and the multi-task intra-day groups has normalized root mean square values (mean \pm std) of $6.8 \pm 2.4,13.2 \pm 3.5$, and $7.0 \pm 2.3$, respectively. For walking at $1.5 \mathrm{~m} / \mathrm{s}$, the single-task, intra-day and single-task, inter-day had normalized root mean square error (NRMSE) values (mean \pm std) of $5.7 \pm 2.1 \%$ and $12.2 \pm 2.3 \%$ respectively and the multi-task, multi-day group had a mean NRMSE value of $6.2 \pm 2.3 \%$. For the calf raises, the single-task, intra-day and single-task, 


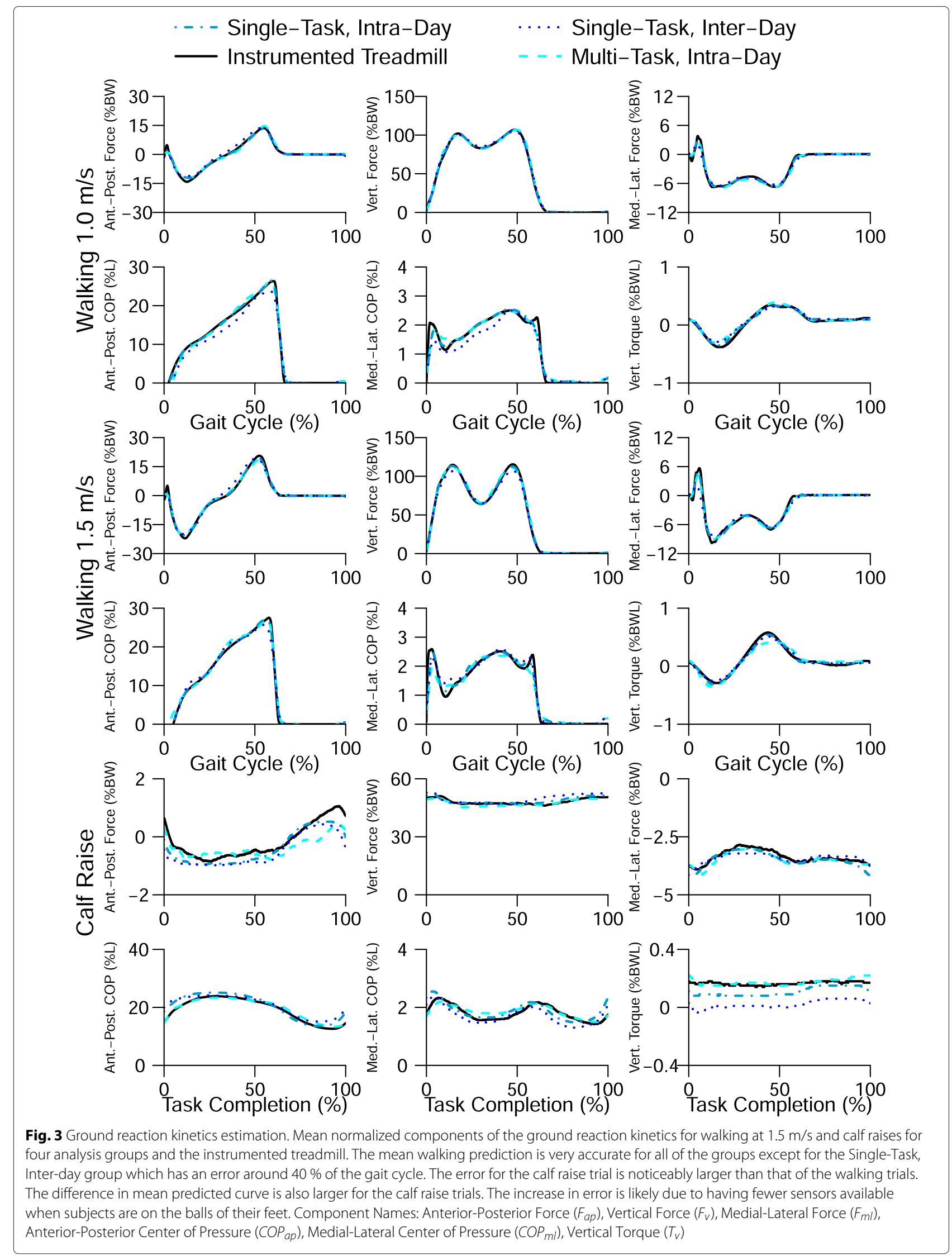




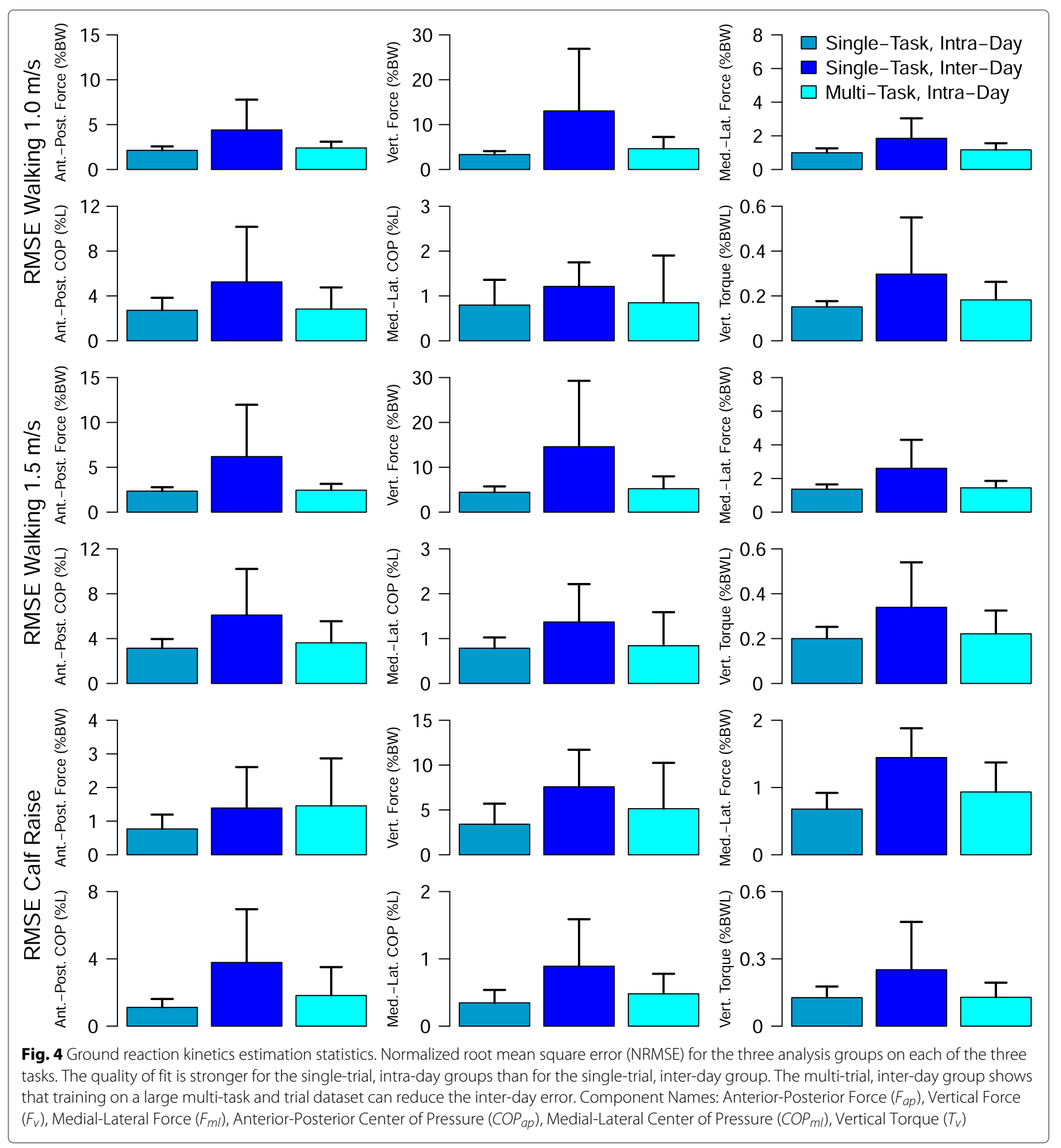

inter-day had NRMSE values (mean \pm std) of $14.2 \pm 5.4 \%$ and $33.3 \pm 8.6 \%$ respectively and the multi-task, multiday group had a mean NRMSE value of $21.7 \pm 9.6 \%$.

\section{Ground reaction kinetics for walking at $1.0 \mathrm{~m} / \mathrm{s}$}

For walking at $1.0 \mathrm{~m} / \mathrm{s}$, the single-task, intra-day model had normalized root mean square error (NRMSE) values less than $10 \%$ for all six components of the ground reaction kinetics (Table 2). The single-task, inter-day model had NRMSE values above $10 \%$ for all six components of the ground reaction kinetics but the three ground reaction forces and the two center of pressure values have NRMSE values (10.9-13.1\%) close to the accuracy criterion. The multi-task, intra-day model has NRMSE values less than $10 \%$ for all of the kinetic components with the exception of the vertical torque $\left(T_{v}\right)$. 
Table 2 Fit metrics for the ground reaction kinetic estimation

\begin{tabular}{|c|c|c|c|c|c|c|}
\hline \multirow[b]{2}{*}{ Walking $1.0 \mathrm{~m} / \mathrm{s}$} & \multicolumn{2}{|c|}{$F_{a p}$} & \multicolumn{2}{|c|}{$F_{v}$} & \multicolumn{2}{|c|}{$F_{m l}$} \\
\hline & RMSE (\% BW) & NRMSE (\%) & RMSE (\% BW) & NRMSE (\%) & RMSE (\% BW) & NRMSE (\%) \\
\hline Single-Task, Intra-Day & 2.13 & 6.21 & 3.33 & 2.93 & 1.00 & 5.82 \\
\hline Single-Task, Inter-Day & 4.40 & 13.07 & 13.06 & 11.48 & 1.85 & 10.97 \\
\hline \multirow[t]{2}{*}{ Multi-Task, Intra-Day } & 2.39 & 6.95 & 4.72 & 4.15 & 1.16 & 6.79 \\
\hline & \multicolumn{2}{|c|}{$C O P_{a p}$} & \multicolumn{2}{|c|}{$C O P_{m l}$} & \multicolumn{2}{|c|}{$T_{v}$} \\
\hline Walking $1.0 \mathrm{~m} / \mathrm{s}$ & RMSE (\% L) & NRMSE (\%) & RMSE (\% L) & NRMSE (\%) & RMSE (\% BWL) & NRMSE (\%) \\
\hline Single-Task, Intra-Day & 2.72 & 5.90 & 0.80 & 7.15 & 0.15 & 9.67 \\
\hline Single-Task, Inter-Day & 5.25 & 10.89 & 1.21 & 12.54 & 0.30 & 20.08 \\
\hline \multirow[t]{2}{*}{ Multi-Task, Intra-Day } & 2.76 & 6.21 & 0.85 & 6.77 & 0.18 & 11.25 \\
\hline & \multicolumn{2}{|c|}{$F_{a p}$} & \multicolumn{2}{|c|}{$F_{v}$} & \multicolumn{2}{|c|}{$F_{m l}$} \\
\hline Walking $1.5 \mathrm{~m} / \mathrm{s}$ & (\% BW) RMSE & (\%) NRMSE & (\% BW) RMSE & (\%) NRMSE & (\% BW) RMSE & (\%) NRMSE \\
\hline Single-Task, Intra-Day & 2.34 & 4.38 & 4.43 & 3.47 & 1.36 & 5.41 \\
\hline Single-Task, Inter-Day & 6.19 & 11.76 & 14.59 & 11.36 & 2.61 & 10.24 \\
\hline \multirow[t]{2}{*}{ Multi-Task, Intra-Day } & 2.53 & 4.74 & 5.37 & 4.19 & 1.44 & 5.69 \\
\hline & \multicolumn{2}{|c|}{$\mathrm{COP}_{a p}$} & \multicolumn{2}{|c|}{$C O P_{m l}$} & \multicolumn{2}{|c|}{$T_{V}$} \\
\hline Walking $1.5 \mathrm{~m} / \mathrm{s}$ & $(\% \mathrm{~L}) \mathrm{RMSE}$ & (\%) NRMSE & $(\% \mathrm{~L}) \mathrm{RMSE}$ & (\%) NRMSE & (\% BWL) RMSE & (\%) NRMSE \\
\hline Single-Task, Intra-Day & 3.14 & 5.25 & 0.79 & 6.60 & 0.20 & 9.35 \\
\hline Single-Task, Inter-Day & 6.10 & 10.57 & 1.37 & 12.84 & 0.34 & 16.50 \\
\hline \multirow[t]{2}{*}{ Multi-Task, Intra-Day } & 3.55 & 5.46 & 0.84 & 6.80 & 0.22 & 10.56 \\
\hline & \multicolumn{2}{|c|}{$F_{a p}$} & \multicolumn{2}{|c|}{$F_{v}$} & \multicolumn{2}{|c|}{$F_{m l}$} \\
\hline Calf Raise & RMSE (\% BW) & NRMSE (\%) & RMSE (\% BW) & NRMSE (\%) & RMSE (\% BW) & NRMSE (\%) \\
\hline Single-Task, Intra-Day & 0.77 & 16.97 & 3.41 & 15.89 & 0.68 & 16.08 \\
\hline Single-Task, Inter-Day & 1.39 & 32.46 & 7.58 & 39.63 & 1.45 & 34.92 \\
\hline \multirow[t]{2}{*}{ Multi-Task, Intra-Day } & 1.57 & 37.22 & 5.33 & 24.98 & 0.91 & 22.48 \\
\hline & \multicolumn{2}{|c|}{$C O P_{a p}$} & \multicolumn{2}{|c|}{$C O P_{m l}$} & \multicolumn{2}{|c|}{$T_{v}$} \\
\hline Calf Raise & RMSE (\% L) & NRMSE (\%) & RMSE (\% L) & NRMSE (\%) & RMSE (\% BWL) & NRMSE (\%) \\
\hline Single-Task, Intra-Day & 1.11 & 5.83 & 0.35 & 9.86 & 0.13 & 20.70 \\
\hline Single-Task, Inter-Day & 3.78 & 20.29 & 0.89 & 28.06 & 0.25 & 44.59 \\
\hline Multi-Task, Intra-Day & 1.78 & 9.19 & 0.49 & 14.32 & 0.13 & 21.74 \\
\hline
\end{tabular}

Root mean squared (RMSE) and Normalized root mean squared error (NRMSE) for the six ground reaction force components of the walking at $1.5 \mathrm{~m} / \mathrm{s}$ trial, and three select of the ground reaction kinetics of the calf raise task. The multi-task, intra-day model performs as well as the single-task, intra-day model which means that with sufficient training data, the insole can be used to study multiple locomotion tasks. Component Names: Anterior-Posterior Force $\left(F_{a p}\right)$, Vertical Force $\left(F_{v}\right)$, Medial-Lateral Force $\left(F_{m l}\right)$, Anterior-Posterior Center of Pressure $\left(C O P_{a p}\right)$, Medial-Lateral Center of Pressure $\left(\mathrm{COP}_{m l}\right)$, Vertical Torque $\left(T_{v}\right)$

\section{Ground reaction kinetics for walking at $1.5 \mathrm{~m} / \mathrm{s}$}

For walking at $1.5 \mathrm{~m} / \mathrm{s}$, the single-task, intra-day model had normalized root mean square error (NRMSE) values less than $10 \%$ for all six components of the ground reaction kinetics (Table 2). The single-task, interday model had NRMSE values above $10 \%$ for all six components of the ground reaction kinetics but the three ground reaction forces and the two center of pressure values have NRMSE values (10.2-12.8\%) close to the accuracy criterion. The multi-task, intraday model has NRMSE values less than $10 \%$ for all of the kinetic components with the exception of the vertical torque.

\section{Ground reaction kinetics for calf raises}

For the calf raise task, the single-task, intra-day model had normalized root mean square error (NRMSE) values less than $10 \%$ for the anterior-posterior center of pressure and medial-lateral center of pressure positions (Table 2). None of the predictions of the single-task, inter-day model were less than $10 \%$. Only the prediction of the anteriorposterior center of pressure was less than $10 \%$ for the multi-task, intra-day model.

\section{Ankle joint torque estimation}

Overall, the insole sensor individually was a stronger predictor of ankle joint torques than the tendon sensor or 
the ankle angle from inverse kinematics. In the multitask, intra-day model, the insole sensor data was sufficient to predict the ankle joint torque for walking at 1.0 and $1.5 \mathrm{~m} / \mathrm{s}$ tasks with normalized root mean square error (NRMSE) values of 8.7 and $9.2 \%$ respectively (Table 3 ). The ankle angle and tendon features, both individually and together, had high normalized root mean square error (NRMSE) values and large deviations in the mean curves (Fig. 5) for all three tasks. Combining the insole data with the ankle angle data or the tendon data resulted in a lower NRMSE on all three tasks. For all tasks, the combination of insole and tendon data produced similar NRMSE as the combination of insole and ankle angle data.

\section{Discussion}

Wearable sensors for estimating the ground reaction forces and the ankle joint moment can provide key biomechanical data for analyzing human motion. Our results show that a low-cost pressure insole and tendon sensor can produce estimates similar to the reported accuracy of commercial devices. For walking, a model produced by training both walking and calf raise data (multi-task, intra-day) produced normalized root mean square error (NRMSE) values for the ground reaction kinetics that were under $10 \%$ for all three components of the force vector and both components of the location of the center of pressure (NRMSE: 4.15-6.80\%). The predictions of the vertical torque (NRMSE: $10.56 \%, 11.25 \%$ ) exceeded the cutoff by a small amount. For the calf raises, only the prediction of the anterior-posterior center of pressure met the accuracy criterion. The results of the prediction of the AJT were similar to the GRF, where the sensor set had accuracy less than $10 \%$ for walking but not for calf raises.

The results of the prototype sensors compare favorably with previous research on walking in commercial sensors
[5, 6, 8]. Font et al. (2008) showed RMSE errors of $5 \%$ for the vertical force, $12 \%$ for the anterior-posterior force, and $28 \%$ for the medial lateral force [6] for walking in the Pedar insoles. Rouhani et al. (2010) also found errors of $4 \%$ for the vertical force, $7.4 \%$ for the anterior-posterior force, $11.3 \%$ for the medial-lateral force, and $14.7 \%$ for the vertical torque in Pedar insoles. In a laboratory prototype, Howell et al. (2012) showed NRMSE of the ankle torque of $5.9 \%$ for their healthy patients and $9.8 \%$ in stroke subjects.

One advantage of the current insole prototype is that the pressure sensors respond to changes in the volume of the air bladder. The capacitive and resistive sensors found in commercial devices are uniaxial only but our prototype sensors produced a signal in response to three dimensional axial or sheer stress. The disadvantage is that the model between sensor voltage and a kinetic component like vertical force is no longer simply linear but our results show that the neural network training is sufficiently accurate.

Our study goes further than previous studies by evaluating our sensor system on a dataset that includes a nonstereotypical motion and multiple testing days. Despite the increased variability in the data set due to the inclusion of inter-day testing and data from both tasks, the multi-task, intra-day, and the single-task, intra-day models had similar accuracy which suggests that the current prototype may be limited by sensor accuracy and not task variability.

Recently Godi et al. (2014), suggested that plantar pressure insoles are potentially more accurate for spatial variables (like peak force and center of pressure) and worse for temporal variables like stance duration especially at lower sampling frequencies [25]. Our high accuracy for vertical force during walking suggests that our system could be used to accuracy predict contact time and stance duration.

Table 3 Fit metrics for the ankle moment estimation

\begin{tabular}{|c|c|c|c|c|c|c|}
\hline \multirow{2}{*}{$\begin{array}{l}\text { Multi-task, Intra-day } \\
\text { ankle joint torque }\end{array}$} & \multicolumn{2}{|c|}{ Calf raise } & \multicolumn{2}{|c|}{ Walking $1.0 \mathrm{~m} / \mathrm{s}$} & \multicolumn{2}{|c|}{ Walking $1.5 \mathrm{~m} / \mathrm{s}$} \\
\hline & RMSE & NRMSE & RMSE & NRMSE & RMSE & NRMSE \\
\hline features & (\% BWL) & (\%) & (\% BWL) & (\%) & (\% BWL) & (\%) \\
\hline Angle & 3.42 & 53.06 & 2.56 & 15.83 & 3.01 & 16.42 \\
\hline Tendon & 3.01 & 46.02 & 3.33 & 20.47 & 4.34 & 23.54 \\
\hline Angle+Tendon & 3.35 & 51.77 & 2.34 & 14.46 & 2.94 & 16.03 \\
\hline Insole & 1.04 & 16.52 & 1.39 & 8.72 & 1.70 & 9.21 \\
\hline Insole+Tendon & 0.90 & 13.78 & 1.17 & 7.42 & 1.30 & 7.04 \\
\hline Angle+Insole & 0.98 & 15.57 & 1.27 & 8.04 & 1.36 & 7.29 \\
\hline Angle+Insole+Tendon & 0.91 & 13.78 & 1.17 & 7.51 & 1.27 & 6.85 \\
\hline
\end{tabular}

Root mean squared error (RMSE) and normalized root mean squared error (NRMSE) for the right ankle moment for the calf raise, walking at $1.0 \mathrm{~m} / \mathrm{s}$, and walking at $1.5 \mathrm{~m} / \mathrm{s}$ trials. The angle and tendon sensors individually have low accuracy (NRMSE > $10 \%$ ) in all three tasks. The insole sensor has high accuracy for the walking tasks but not for the calf raise tasks. Combining redundant measurements from the insole sensor and the tendon sensor improves accuracy on all three tasks. Units: Body Weight * Leg Length (BWL) 


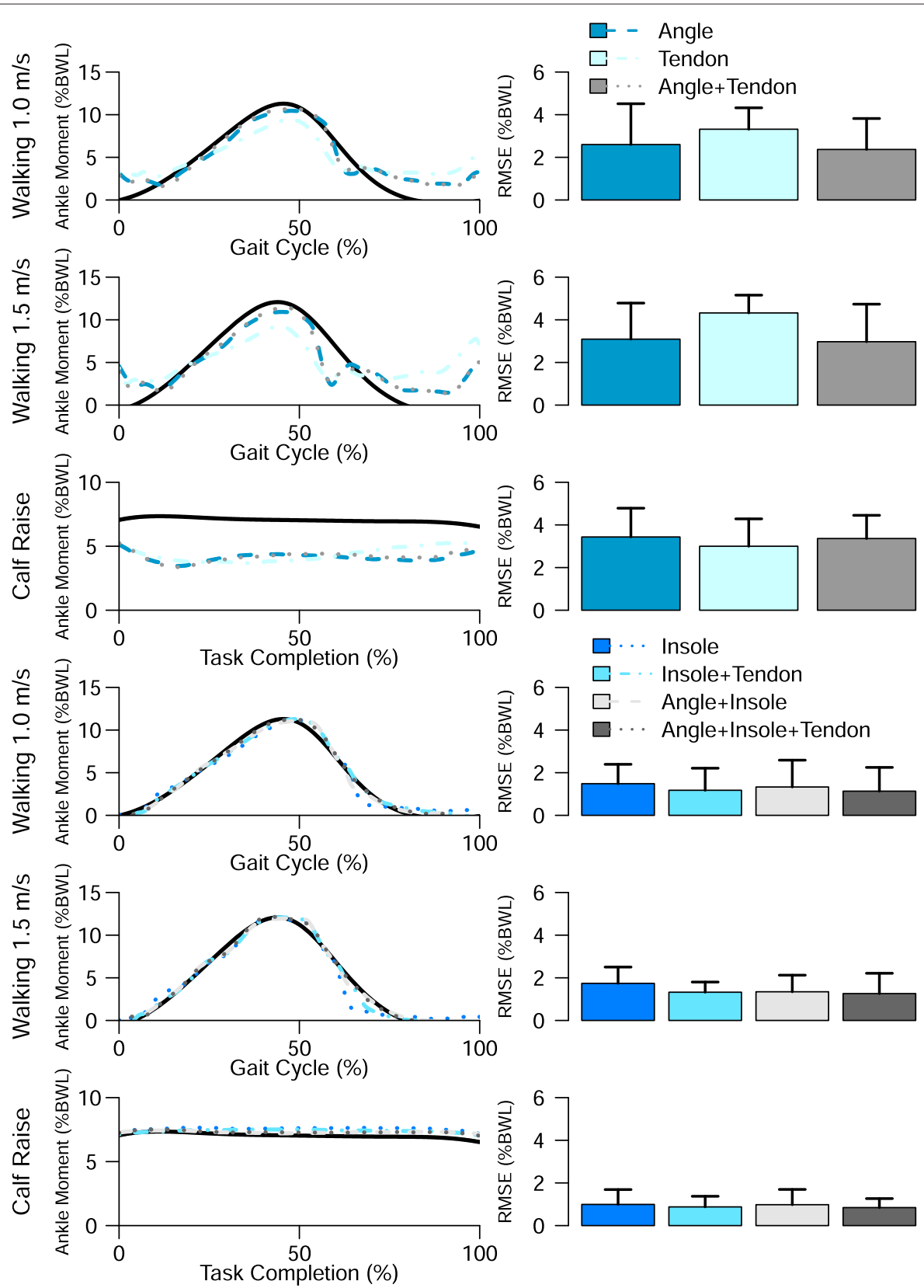

Fig. 5 (Top) Mean normalized right ankle moments as calculated by Inverse Dynamics and estimated by the multi-task, multi-day model for each of the seven sensor combinations on the three tasks. The angle and tendon sensors are not sufficient to predict moment for all of the tasks by themselves. (Bottom) Normalized root mean squared error (NRMSE) for the seven sensor combinations on the three different tasks. The insole sensor alone outperforms the all of the other sensors individually. Combining the angle and tendon sensor with the insole has a small benefit for the NRMSE

For the ankle joint torque estimations, we found that generally the insole data was the more accurate than the ankle angle from inverse kinematics or the tendon sensor. The insole data alone were accurate for walking (RMSE $\leq 10 \%$ ) but insufficient for the calf raise task. Including, the tendon sensor, which had a lower individual NRMSE value than the insole sensor, lowered the NRMSE value for both the calf raise and the walking tasks. With both the insole and tendon sensor as features, the addition of the ankle angle calculated from inverse kinematics did not substantially improve the NRMSE value. The ankle joint torque predictions show the beneficial strategy of including sensor redundancy in estimation.

Our results demonstrate that estimating the calf raise task was particularly difficult for the prototype sensors. The plantar pressure insole predicted the majority of the 
ground reaction forces data for walking and but only very little for calf raises. One limitation of the prototype insole sensor is that the spatial resolution is low. During the calf raise task, the majority of the motion is spent balancing on the forefoot where only 5 of the 8 sensors are active which further decreases the spatial resolution of the signals. One way to improve the estimation algorithms across these dissimilar tasks would be to include knowledge of foot state in order to trigger different stored models.

Most subjects did not report any major comfort issues during the performance of the tasks. A few of the shorter subjects did complain that the tendon sensor was uncomfortable around the moment of peak calf raise height. An important consideration for at-home health monitoring systems is the burden of the system on the the likelihood of adoption and continued use by patients. Future work on the prototype sensors will focus on further reducing the weight and increasing the comfort of the sensors. Our prototype is also currently limited by the wired connection to the data acquisition system. However, the sensors all return voltage signals which means that an off-the-shelf microcontroller with an analog to digital converter component and a wireless emitter could readily be used to read in the sensor data.

Another point of future work will be to develop a proper set of stereotypical and non-stereotypical tasks to act as a calibration set for true at home monitoring. While calfraises are an interesting balancing task, movements that are part of the activities of daily living such as reaching for items, turning and sit-to-stand transfers are important elements of future research.

\section{Conclusion}

We developed a sensing system of two low-cost sensors for estimating the ground reaction forces and ankle joint torque of a data set which include walking at $1.0 \mathrm{~m} / \mathrm{s}$, walking at $1.5 \mathrm{~m} / \mathrm{s}$, and calf raises on multiple collection days. A multi-task, intra-day model was able to accurately predict the ground reaction forces and ankle joint torque at both walking speeds. The combination of the insole and the tendon sensor produced more accurate predictions than the individual sensors in both the walking and the calf raise tasks. Estimates of the ground reaction kinetics and ankle joint torque on the calf raise task were worse than walking which suggests task-specific deficiencies that should be further studied.

\section{Competing interests}

The authors declare that they have no competing interests.

\section{Authors' contributions}

DJ and DF designed the research study. DJ designed the sensing hardware, performed the data collection, analyzed the data. DJ and DF interpreted the data and drafted the manuscript. DJ and DF read and approved the final manuscript.

\section{Acknowledgments}

This research was funded by National Science Foundation (IIP-1026872) and by the Department of Defense (W81XWH-09-2-0142).

Received: 2 June 2015 Accepted: 29 September 2015

Published online: 14 October 2015

\section{References}

1. Baig MM, Gholamhosseini H. Smart health monitoring systems: an overview of design and modeling. J Med Syst. 2013. 37(2).

2. Pantelopoulos A, Bourbakis NG. A survey on wearable sensor-based systems for health monitoring and prognosis. IEEE Trans Syst Man Cybern Part C Appl Rev. 2010;40(1):1-12. doi:10.1109/TSMCC.2009.2032660.

3. Park S, Jayaraman S. Enhancing the quality of life through wearable technology. IEEE Eng Med Bio Mag. 2015;22(3):41-8. doi:10.1109/MEMB.2003.1213625.

4. Chesnin KJ, Selby-Silverstein L, Besser MP. Comparison of an in-shoe pressure measurement device to a force plate: concurrent validity of center of pressure measurements. Gait \& Posture. 2000;12(2):128-33. doi:10.1016/S0966-6362(00)00071-0. Accessed 06 Nov 2013.

5. Forner Cordero A, Koopman HJFM, van der Helm FCT. Use of pressure insoles to calculate the complete ground reaction forces. J Biomech. 2004;37(9):1427-32. doi:10.1016/j.jbiomech.2003.12.016. Accessed 07 Oct 2013.

6. Fong DT-P, Chan YY, Hong Y, Yung PS-H, Fung KY, Chan K-M, et al. Estimating the complete ground reaction forces with pressure insoles in walking. J Biomech. 2008;41(11):2597-601. doi:10.1016/j.jbiomech.2008. 05.007. Accessed 15 Jul 2013.

7. Liu T, Inoue Y, Shibata K. A wearable force plate system for the continuous measurement of triaxial ground reaction force in biomechanical applications. Meas Sci Technol. 2010;21(8):085804. doi:10.1088/0957-0233/21/8/085804. Accessed 25 Jul 2013.

8. Howell A, Kobayashi T, Hayes H, Foreman K, Bamberg S. Kinetic gait analysis using a low-cost insole. IEEE Trans Biomed Eng. 2013. Early Access Online: doi:10.1109/TBME.2013.2250972.

9. Liedtke C, Fokkenrood SAW, Menger JT, van der Kooij H, Veltink PH. Evaluation of instrumented shoes for ambulatory assessment of ground reaction forces. Gait \& Posture. 2007;26(1):39-47. doi:10.1016/j.gaitpost.2006.07.017. Accessed 15 Jul 2013.

10. Rouhani H, Favre J, Crevoisier X, Aminian K. Ambulatory assessment of $3 \mathrm{~d}$ ground reaction force using plantar pressure distribution. Gait \& Posture. 2010;32(3):311-6. doi:10.1016/j.gaitpost.2010.05.014. Accessed 07 Oct 2013.

11. Silbernagel KG, Steele R, Manal K. Deficits in heel-rise height and achilles tendon elongation occur in patients recovering from an achilles tendon rupture. Am J Sports Med. 2012;40(7):1564-71. doi:10.1177/0363546512447926. Accessed 08 Sept 2015.

12. Ockuly A, O'Brien L, LaPrade RF. Rehabilitation of complex knee injuries and key points In: Doral MN, Karlsson J, editors. Sports injuries. Berlin, Heidelberg: Springer. p. 1467-77.

13. Williams SB, Brand CA, Hill KD, Hunt SB, Moran H. Feasibility and outcomes of a home-based exercise program on improving balance and gait stability in women with lower-limb osteoarthritis or rheumatoid arthritis: a pilot study. Arch Phys Med Rehabil. 1976;91(1):106-14. doi:10.1016/j.apmr.2009.08.150. Accessed 08 Sept 2015.

14. Barnes $G R G$, Pinder DN. In vivo tendon tension and bone strain measurement and correlation. J Biomech. 1974;7(1):35-42. doi:10.1016/0021-9290(74)90068-2. Accessed 29 Nov 2014.

15. Hahs DW, Stiles RN. Buckle muscle tension transducer: What does it measure? J Biomech. 1989;22(2):165-6. doi:10.1016/0021-9290(89)90039-0. Accessed 29 Nov 2014.

16. Kots YAM, Absalyamov TM, Zorin VP, Koryak Y, Kuznetsov SP, Sin LD. Modification of the tendometeric method measuring a single human muscles response the force. Hum Physiol. 1976;2:1046-1048.

17. Pourcelot $P$, Defontaine $M$, Ravary B, Lemâtre $M$, Crevier-Denoix N. A non-invasive method of tendon force measurement. J Biomecha. 2005;38(10):2124-129. doi:10.1016/j.jbiomech.2004.09.012. Accessed 11 Aug 2014

18. Cavanagh PR, Hewitt Jr FG, Perry JE. In-shoe plantar pressure measurement: a review. The Foot. 1992;2(4):185-94. doi:10.1016/09582592(92)90047-S. 06 Nov 2013. 
19. Rosenbaum D, Becker HP. Plantar pressure distribution measurements. Technical background and clinical applications. Foot Ankle Surg. 1997;3(1):1-14. doi:10.1046/j.1460-9584.1997.00043.x. Accessed 07 Nov 2014.

20. Abdul Razak AH, Zayegh A, Begg RK, Wahab Y. Foot plantar pressure measurement system: a review. Sensors. 2012;12(7):9884-912. doi:10.3390 /s120709884.07 Nov 2014.

21. Hurkmans HLP, Bussmann JBJ, Benda E, Verhaar JAN, Stam HJ. Accuracy and repeatability of the Pedar mobile system in long-term vertical force measurements. Gait \& Posture. 2006;23(1):118-25. doi:10.1016/j.gaitpost.2005.05.008. Accessed 22 Sept 2014.

22. Ramanathan AK, Kiran P, Arnold GP, Wang W, Abboud RJ. Repeatability of the Pedar- $X{ }^{\circledR}$ in-shoe pressure measuring system. Foot Ankle Surg. 2010;16(2):70-3. doi:10.1016/j.fas.2009.05.006. Accessed 22 Sept 2014

23. Arndt A. Correction for sensor creep in the evaluation of long-term plantar pressure data. J Biomech. 2003;36(12):1813-17. doi:10.1016/S0021-9290(03)00229-X. Accessed 22 Sept 2014.

24. Giacomozzi C. Appropriateness of plantar pressure measurement devices: a comparative technical assessment. Gait \& Posture. 2010;32(1):141-4. doi:10.1016/j.gaitpost.2010.03.014. Accessed 07 Nov 2014.

25. Godi M, Turcato AM, Schieppati M, Nardone A. Test-retest reliability of an insole plantar pressure system to assess gait along linear and curved trajectories. J NeuroEngineering Rehabil. 2014;11(1):95. doi:10.1186/ 1743-0003-11-95. Accessed 14 Jan 2015.

26. Hurkmans HLP, Bussmann JBJ, Selles RW, Horemans HLD, Benda E, Stam HJ, et al. Validity of the Pedar mobile system for vertical force measurement during a seven-hour period. J Biomech. 2006;39(1):110-8. doi:J10.1016/j.jbiomech.2004.10.028. Accessed 09 Apr 2015.

27. Delp SL, Anderson FC, Arnold AS, Loan P, Habib A, John CT, et al. OpenSim: open-source software to create and analyze dynamic simulations of movement. IEEE Trans Biomed Eng. 2007;54(11):1940-50. doi:10.1109/TBME.2007.901024.

28. Friedman J, Hastie T, Tibshirani R. The elements of statistical learning, $2^{\text {nd }}$ ed., Springer Series in Statistics. New York: Springer-Verlag; 2009. doi:10.1007/978-0-387-84858-7.

29. Picard RR, Cook RD. Cross-validation of regression models. J Am Stat Assoc. 1984;79(387):575. doi:10.2307/2288403. 10 Sept 2015.

\section{Submit your next manuscript to BioMed Central} and take full advantage of:

- Convenient online submission

- Thorough peer review

- No space constraints or color figure charges

- Immediate publication on acceptance

- Inclusion in PubMed, CAS, Scopus and Google Scholar

- Research which is freely available for redistribution

Submit your manuscript at www.biomedcentral.com/submit
C BiolMed Central 